\title{
Electrical Properties of CdS/Polyaniline Heterojunction
}

\author{
D. Patidar, N. Jain, N.S. Saxena, Kananbala Sharma, and T.P. Sharma \\ Semiconductor and Polymer Science Laboratory, Department of Physics, \\ 5-6, Vigyan Bhawan, University of Rajasthan, Jaipur-302004, India
}

Received on 4 January, 2006

\begin{abstract}
Conducting polyaniline (PANI) has been synthesized using Sol-gel technique with chemical oxidation process. Chemically prepared cadmium sulphide has been printed on pellet of conducting polyaniline using screen-printing technique. I-V characteristics of conducting polyaniline and CdS printed conducting polyaniline have been recorded at room temperature using Keithley Electrometer. The results indicated that I-V of conducting polyaniline shows non-ohmic behaviour and I-V characteristic of CdS printed PANI, a CdS/ PANI heterojunction, shows the rectification effect and confirms that a diode can be fabricated by using simple technique.
\end{abstract}

Keywords: Polyaniline; Cadmium sulphide; Non-ohmic behaviour; Heterojunction

\section{INTRODUCTION}

In the last two decades conducting polymers have emerged as a new class of materials for their applications in advanced technology and electronic devices. Devices based on conducting polymers have demonstrated potential for application such as sensing by detecting vapour of variety of gases [1,2], lightweight batteries [3], Schottky diodes [4], field effect transistors [5] and light emitting diodes [6]. The electrical conductivity of conducting polymers can be changed from insulating to metallic by chemical or electrochemical doping, which is related to the redox state (doping level) of polymer. In addition to this, other different properties of conducting polymer could be modified by their interaction with other substances.

Among the family of conducting polymers, polyaniline (PANI) is the most suitable for development of new devices because its electrical properties can be changed either by oxidation of the polymer chains or via protonation of the imine nitrogen backbone. Besides other properties, it also presents thermal and environmental stability.

Rectifying junctions are the basic elements of many electronic components. Since the discovery of the conducting polymers rectifying junction such as p-n junction and Schottky junction have been studied so as to explore the possible application of conducting polymers. However, a stable p-n junction could not be fabricated successfully from semiconducting polymers because $\mathrm{p}$ - and n-types of doping cannot be separately introduced to neighbouring layers. This problem can be overcome by attempting the fabrication of conducting polymer-based junction of semiconductor and conducting polymer or Schottky junction between conducting polymer and metal.

Early studies related to organic-inorganic junction generally involved polypyrrole (PPy) and bulk cadmium sulphide (CdS) [7], polyphenylene vinylene (PPv) and CdS films [8] and PANI and Silicon [9]. However, to the best of our knowledge no attempt has been made to investigate the behaviour of heterojunctions formed from PANI and CdS. From this point of view, an effort has been made to develop a new process to produce polyaniline-CdS heterojunction diodes and examine the performance of such a device.
In this article, an attempt has been made to discuss the preparation of conducting polyaniline by using Sol-Gel method. Chemically precipitated $\mathrm{CdS}$ has been printed using screen-printing technique on the pellet of PANI so as to fabricate a heterojunction of PANI and CdS. The I-V characteristics of PANI and CdS/PANI heterojunction have been determined by Keithley Electrometer/ High Resistance meter $6517 \mathrm{~A}$ at room temperature.

\section{MATERIAL PREPARATION}

Polyaniline has been synthesized using chemical oxidation process of aniline monomer with ammonium peroxydisulfate as oxidant. For this purpose, double distilled aniline (.01M) is dissolved in precooled $\mathrm{HCl}(1 \mathrm{M})$ solution. A calculated amount of ammonium peroxydisulphate $(.01 \mathrm{M})$ is dissolved in $\mathrm{HCl}(1 \mathrm{M})$, which has already been cooled to $0^{\circ} \mathrm{C}$. Now this aqueous solution of ammonium peroxydisulphate is added to the stirred solution of aniline dissolved in $\mathrm{HCl}$. The temperature of this solution was maintained at $3-4{ }^{\circ} \mathrm{C}$ during the time of mixing. The dark green precipitate resulting from this reaction is kept in $\mathrm{HCl}$ solution for 24 hours. After keeping the precipitate overnight, it was washed with distilled water and methanol. Now this precipitate is dried under dynamic vacuum at $60{ }^{\circ} \mathrm{C}$ temp, to obtain PANI in the salt form [10]. Pellets of conducting polyaniline (salt) have been prepared using pressure machine at a load of 5 tons. Thickness and diameter of the pellets of PANI are $2 \mathrm{~mm}$ and $12 \mathrm{~mm}$.

$\mathrm{CdS}$ has been prepared by chemical method using $\mathrm{Cd}, \mathrm{HCl}$ $\& \mathrm{H}_{2} \mathrm{~S}$. Cadmium metal was completely dissolved in $\mathrm{HCl}$ solution. Then $\mathrm{H}_{2} \mathrm{~S}$ gas was passed through the solution. The yellow precipitate obtained in the above process was filtered. This precipitate was then dried in an oven at $50{ }^{\circ} \mathrm{C}$ for 30 minutes. When the precipitate was completely dried, it was then crushed to fine powder by grinding process.

$\mathrm{CdS}$ has been printed on PANI using screen-printing technique [11]. The paste of CdS for screen-printing was prepared by using cadmium chloride as an adhesive source and ethylene glycol as a binder. The weight of cadmium chloride was only $10 \%$ of the weight of CdS. The paste was then screen printed 
on pellet of polyaniline. CdS printed on PANI pellet was then sintered in an oven at $150{ }^{\circ} \mathrm{C}$ for six hours for ensuring a good adhesion.

\section{RESULTS \& DISCUSSION}

The current-voltage characteristic of conducting polyaniline and CdS/PANI heterojunction were determined by applying the silver paste contact on both side of samples at room temperature using Keithley Electrometer/High Resistance meter $6517 \mathrm{~A}$. This equipment has an in built capacity of output independent voltage source of $\pm 1000 \mathrm{~V}$. We apply the voltage across the sample to measure the current through the sample.

Figure 1 shows the I-V characteristic of polyaniline bulk (pellet) at room temperature. It is observed that $\mathrm{I}-\mathrm{V}$ characteristic of polyaniline is completely symmetrical with respect to the polarity of the applied voltage. The curve follows nonohmic relation. This non-linear increase in current with applied voltage is explained by the conduction mechanism of polyaniline. Here charge conduction is not only carried by free carriers (electron \& hole) such as in intrinsic semiconductors but also is carried by the formation of polarons and bipolarons. As the applied voltage is increased the formation of polarons and bipolarons increase rapidly contributing to higher values of current through the sample.

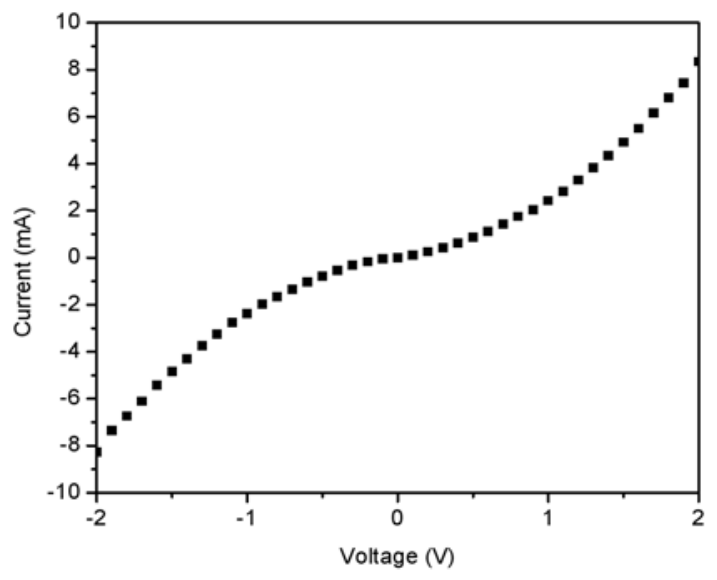

FIG. 1: I-V characteristic of PANI.

The current-voltage characteristic of CdS/PANI heterojunction was taken at room temperature as shown in Fig. 2 and indicates that cadmium sulphide coated on polyaniline pellet behave as Schottky junction. The forward bias corresponds to the positive potential to the polyaniline with respect to $\mathrm{CdS}$. The I-V characteristic of this junction shows the rectification effect, which indicates the formation of the depletion layer at CdS:PANI interface. The Schottky equation, which describes the evaluation of current as a function of the applied voltage is given below:

$$
I=I_{S}\left[\exp \left(e V / n k_{B} T\right)-1\right]
$$

and

$$
I_{s}=A^{*} T^{2} \exp \left(-e \Phi_{b} / k_{B} T\right)
$$

where $\mathrm{e}$ is the charge of electron, $\mathrm{V}$ is the applied voltage, $\mathrm{n}$ is the diode ideality factor, $\mathrm{k}_{B}$ is the Boltzmann constant, $\mathrm{T}$ is the temperature, $\Phi_{b}$ is effective barrier height, $A^{*}$ is effective Richardson constant and $\mathrm{I}_{s}$ is the reverse saturation current.

From Fig. 2, it is observed that I-V characteristic of CdS/PANI heterojunction shows the rectification effect. A space charge layer is created at the CdS/PANI interface. The $\mathrm{I}-\mathrm{V}$ characteristic of CdS/PANI heterojunction under the forward bias condition shows the exponential rise at low voltage. This is due to decrease in the width of depletion layer at the junction. At higher voltages the I-V dependence is almost linear because the depletion layer is minimized at the interface and bulk PANI behave as series resistance in the junction. Series resistance has been determined from the I-V characteristic of the junction in the higher region and is found to be $1.09 \mathrm{k} \Omega$.

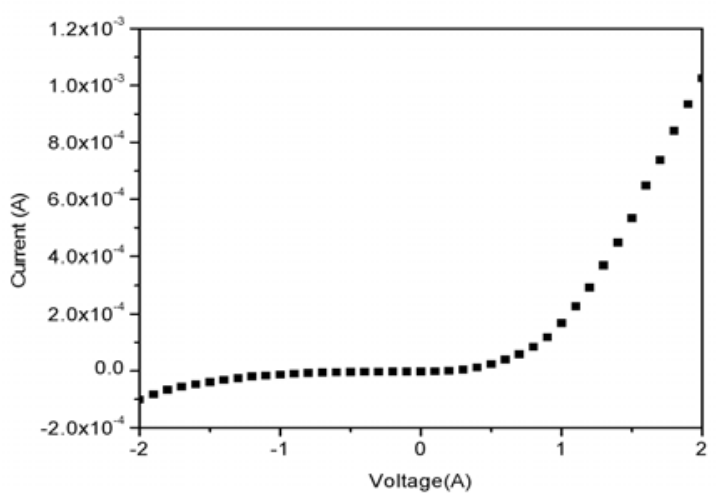

FIG. 2: I-V characteristic of CdS/PANI heterojunction.

In the reverse bias the depletion layer increases due to introduction of high barrier potential and almost all the current is just because of minority charge carriers of $\mathrm{CdS}$. This produces fairly small current in the junction.

The reverse saturation current $\left(\mathrm{I}_{S}\right)$ is determined by interpolation of exponential slope of $\mathrm{I}$ at $\mathrm{V}=0$ and the value of diode ideality factor has been calculated using Eq. 1. It is found that the reverse saturation current $\left(\mathrm{I}_{S}\right)$ is $2.3473 \times 10^{-6} \mathrm{~A}$ and diode ideality factor is 1.33 . This value is in good agreement with the values obtained by other workers [8]. The value of ideality factor greater than unity can be attributed to the recombination of electrons and holes in the depletion region and it is also associated with Fermi-level pinning at the interface, or with a relatively large voltage drops in interface region. The Schottky barrier height $\left(\Phi_{b}\right)$ has also been determined from Eq. (2), which is $0.55 \mathrm{~V}$. It is observed that the Schottky barrier effect disappears at higher voltage beyond which the current is mainly contributed from bulk resistance of the polyaniline. It is noted that in polyaniline devices the $\mathrm{CdS} / \mathrm{PANI}$ interface must be responsible for the rectifying behavior. 


\section{CONCLUSIONS}

As a brief summary of the paper, an organic-inorganic heterojunction diode based on polyaniline and $\mathrm{CdS}$ has been fabricated using screen-printing technique. The currentvoltage characteristic of this junction performs rectifying effect, which is interpreted in terms of barrier formation at the CdS/PANI interface.

\section{Acknowledgements}

One of the authors (Dinesh Patidar) is grateful to DRDO, Govt of India, New Delhi for providing Junior Research Fellowship. Author would like to thank Mr. Vibhav Saraswat, Mr. Vimal Kishore, Ms. Vinodini Saktawat and Mrs. Rashmi Saxena for help in various ways during the course of this work.
[1] M. Wolszczak, J. Kroh, and M. M. Abdel-Hamid, Radiate Phys. Chem. 45, 71 (1995).

[2] J. M. G. laranjeira, H. J. Khoury, W. M. de Azevedo, E. F. da Silva Jr., and E. A. da Vasconcelos, Braz. J. Phys. 32, 1 (2002)

[3] E. Kalayclooglu, U. Akbulut, and L. Toppare, J. Appl. Polym. Sci. 61, 1067 (1996).

[4] H. Koezuka, A. Tsumura, Synthetic Metals 28, C-753 (1989).

[5] G. Gustafsson, G. M. Treacy, Y. Cao, and A. J. Heeger, Synthetic Metals 55-57, 4124 (1993).

[6] J. C. Chiang, A. G. Macdiarmid, Synthetic Metals 13, 193 (1986).

[7] D. Tsamouras, E. Dalas, S. Sakkopoulos, and E. Vitorotos,
Appl. Surf. Sci. 65-66, 388 (1993).

[8] N. D. Kumar, M. P. Joshi, C. S. Ftriend, P. N. Prasad, and R. Burzynshi, Appl. Phy. Lett. 71, 1388 (1997).

[9] J. M. G. Laranjeira, H. J. Khoury, W. M. de Azevedo, E. F. da Silva Jr., and E. A. da Vasconcelos, Appl. Surf. Sci. 190, 390 (2002).

[10] H. S. Nalwa, Handbook of Organic Conductive Molecules \& Polymers, John Wiley \& Sons Ltd. Vol. 2 (1997).

[11] L. Kumar, B. P. Singh, A. Misra, S. C. K. Misra, and T. P. Sharma, Physica B 363, 102 (2005). 